\title{
Point of Care Ultrasound for the General Internist: Pleural Effusions
}

\author{
By Darrel Cotton, Ryan Lenz, Brendan Kerr, Irene Ma
}

DOI: $10.22374 /$ cjgim.v13i2.231

\begin{abstract}
About the Authors
Darrel Cotton is clinical associate professor Department of Medicine Division of General Internal Medicine University of Calgary Ryan Lenz is clinical lecturer Department of Medicine Division of General Internal Medicine University of Calgary

Brendan Kerr is clinical assistant professor Department of Medicine Division of General Internal Medicine University of Calgary Irene Ma is assistant professor Department of Medicine Division of General Internal Medicine University of Calgary Corresponding Author:dwcotton@ucalgary.ca

Submitted: August 8, 2017. November 10, 2017. Published June 25, 2018.
\end{abstract}

\begin{abstract}
Pleural effusions are a common finding in many clinical settings and have important diagnostic and therapeutic implications. They may be identified by physical exam, chest radiography, chest computerized tomography (CT) scans or point of care ultrasonography (POCUS). The use of POCUS for the diagnosis and management of pleural effusions offers several advantages relevant to the practice of the general internist. POCUS in-fact has superior sensitivity and specificity for locating pleural effusions, when compared to chest radiography and physical exam. Abnormal sonographic features of the pleural fluid and the adjacent pleura may also suggest the presence of an exudative or malignant effusion. POCUS additionally can be used to quickly estimate the size of a pleural effusion. Lastly, the use of ultrasound guidance when performing a thoracentesis reduces the risk of a pneumothorax and/or hemorrhage.

\section{RESUME}

Les épanchements pleuraux sont courants dans de nombreux contextes cliniques et ont des implications diagnostiques et thérapeutiques importantes. Ils peuvent être identifiés par un examen physique, une radiographie thoracique, une tomodensitométrie thoracique (TDM) ou une échographie au point de service (POCUS). L’utilisation de POCUS pour le diagnostic et la gestion des épanchements pleuraux offre plusieurs avantages pertinents pour la pratique de l'interniste général. En fait, POCUS a une sensibilité et une spécificité supérieures pour localiser les épanchements pleuraux, en comparaison avec la radiographie thoracique et l'examen physique. Les caractéristiques échographiques anormales du liquide pleural et de la plèvre adjacente peuvent également suggérer la présence d'un épanchement exsudatif ou malin. POCUS peut en outre être utilisé pour estimer rapidement la taille d'un épanchement pleural. Enfin, l'utilisation du guidage échographique lors d'une thoracentèse réduit le risque de pneumothorax et/ou d'hémorragie.
\end{abstract}

Pleural effusions are a common finding in many clinical settings and have important implications for diagnosis and management. The incidence of pleural effusion on medical wards ranges from $16-62 \% .{ }^{1}$ A pleural effusion may be identified by physical exam, chest radiography, chest computerized tomography (CT) scans, POCUS or a combination thereof. The most common test currently used is the standard two-view chest radiograph. It is widely available and convenient to obtain; however standard views can be time consuming and logistically challenging to obtain in acutely ill medical inpatients. In these circumstances, 
physicians rely on a single supine anterior-posterior chest radiograph to help guide management, which can lead to a missed or misdiagnosed pleural effusion. ${ }^{2}$

Thoracic ultrasonography has been available since the 1960s but has lacked widespread adoption due to the comparative ease and low cost of chest radiography. With recent improvements in ultrasound portability and cost, the detection of pleural effusion with bedside or point of care ultrasonography (POCUS) has become more common in clinical practice in multiple areas of medicine.

POCUS offers fast, accurate detection of pleural effusions and reduces the complications associated with thoracentesis. ${ }^{3,4}$ In this article, we summarize the evidence supporting the use of POCUS for the diagnosis and management of pleural effusions as relevant to the practice of the general internist.

\section{Diagnostic Performance of Ultrasound}

For the diagnosis of pleural effusions, POCUS has superior sensitivity and specificity when compared to chest radiography and physical exam. To better understand this, it is worthwhile to briefly review how a pleural effusion is diagnosed using ultrasound.

Ultrasound images are typically acquired with a patient in a sitting or supine position. Diagnosis requires the identification of fluid within the pleural space, which typically (but not always) appearing as an anechoic (black) area between diaphragm, lung and chest wall (Figure 1). Crucial to confirming the presence of a pleural effusion is additionally identifying the thoracic spine. In contrast to chest radiography, in which structures of the thorax are superimposed upon on another; ultrasound images are acquired in a transverse or sagittal plane, making the identification of pleural fluid comparatively easy for even small volumes of pleural fluid. If chest CT is used as the gold standard, POCUS has superior test characteristics compared to chest radiographs, primarily due to a markedly greater sensitivity (sensitivity $94 \%$ vs. $51 \%$, specificity $98 \%$ vs. $91 \%$, respectively). ${ }^{5}$ Despite technical limitations, pocket-sized ultrasound devices have also demonstrated similar superiority in comparison to supine chest radiography (sensitivity $91 \%$ vs. $74 \%$, specificity $100 \%$ vs. $32 \%$, respectively). ${ }^{6}$ In one study, with minimal 3 hours of focused training, novice residents were able to achieve excellent inter-rater reliability compared with experts. ${ }^{7}$

In contrast, physical exam has only moderate sensitivity and specificity when compared to chest radiography for this purpose (sensitivity $73 \%$, specificity $74 \%$ ). ${ }^{8}$ Compared to CT scans as the reference standard, diagnostic accuracy of POCUS also outperformed chest radiograph and auscultation for the detection of pleural effusion in critically ill patients (diagnostic accuracy of $93 \%, 47 \%$, and $61 \%$, respectively). ${ }^{9}$

Using an ultrasound probe in diagnosing pleural effusions, a General Internist undoubtedly has a number of significant advantages. As summarized in Table 1, ultrasound has superior sensitivity and specificity in detecting pleural effusions when compared to physical exam or chest radiography. Furthermore, a focused ultrasound examination of the lungs, in experienced hands can be performed and interpreted within minutes at the

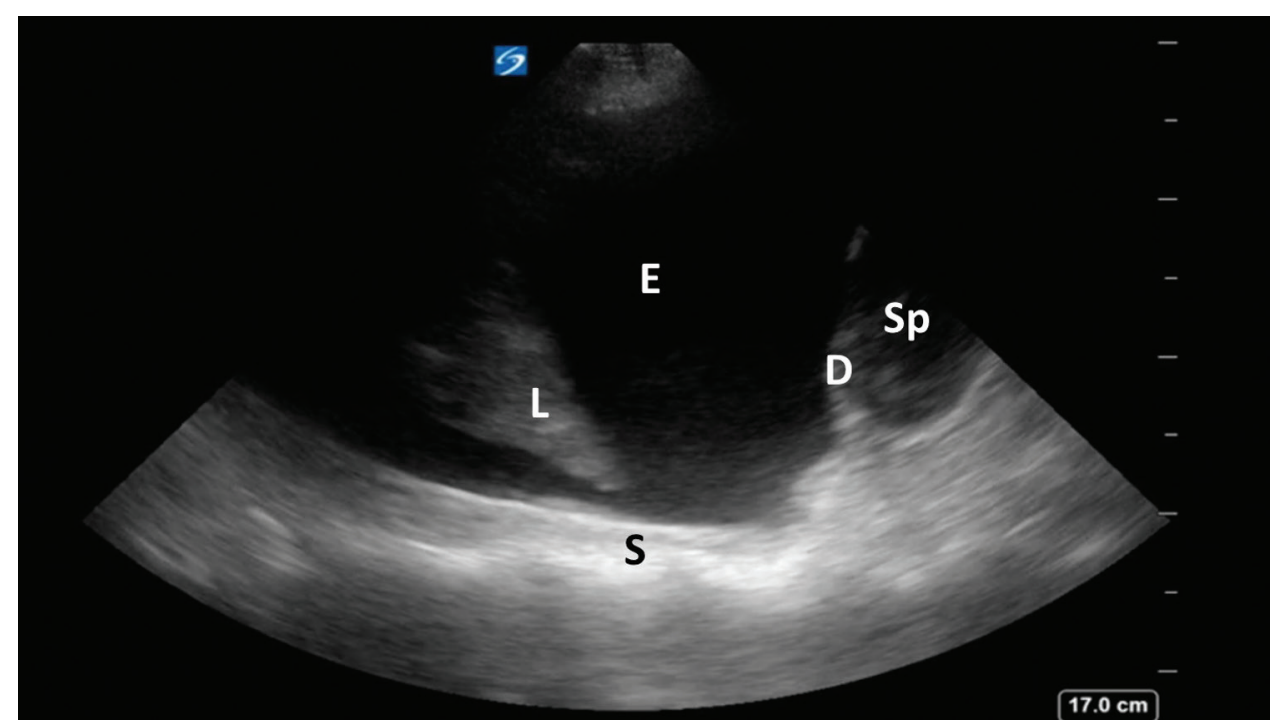

Figure 1. A sagittal ultrasound image of a pleural effusion (E), with lung tip (L), diaphragm (D), spine (S), and spleen (Sp) identified. The pleural effusion appears anechoic (black). Depth is displayed on the right of the image in centimeters. 
bedside. ${ }^{10}$ Lastly, differential diagnostic considerations on chest radiography such as consolidation, atelectasis and pleural disease are also often easily visualized by ultrasound. ${ }^{2}$

\section{Exudative and Malignant Effusions}

An additional advantage of POCUS is that it is also able to detect features of exudative and malignant pleural effusions. These signs of an exudative and malignant effusion are often reflected in sonographic abnormalities of the pleural fluid and pleura.

The sonographic appearance of pleural fluid can be divided into four patterns: anechoic (see Figure 1), complex non-septated (Figure 2), complex septated (Figure 3) and homogenously echogenic (Figure 4). ${ }^{11,12}$ Of these patterns, the anechoic pattern is commonly encountered in clinical practice and is seen in both in exudative and transudative effusions. ${ }^{11}$ It is also the pattern that is most easily identified. The remaining three sonographic patterns are highly suggestive of exudative effusions, of which some may be malignant in origin.

A complex septated pattern is defined by the presence of septations and/or fibrin strands within the pleural fluid and suggests an exudative effusion. A complex non-septated pattern is characterized by the presence of mobile echogenic particles or debris within the pleural fluid; it has also been referred to a swirl sign or plankton sign. This pattern has traditionally been thought to be only found in exudative pleural effusions, however a previous retrospective study found this pattern present in 55\%

Table 1. Diagnostic Performance of Physical Exam, Chest Radiography and Ultrasound for the Diagnosis of Pleural Effusion

\begin{tabular}{|c|c|c|c|c|}
\hline Physical Exam & Sensitivity & Specificity & $+\mathrm{LR}$ & -LR \\
\hline Asymmetric Chest Expansion ${ }^{8}$ & 74 & 91 & 8.1 & 0.29 \\
\hline Dullness $^{8}$ & 73 & 91 & 8.7 & 0.31 \\
\hline \multicolumn{5}{|l|}{ Imaging } \\
\hline $\mathrm{CXR}^{5}$ & 51 & 91 & 5.7 & 0.54 \\
\hline$U S^{5}$ & 94 & 98 & 47 & 0.06 \\
\hline
\end{tabular}

$+\mathrm{LR}=$ positive likelihood ratio; $-\mathrm{LR}=$ negative likelihood ratio; $C X R=$ chest radiograph; $U S=$ ultrasound.

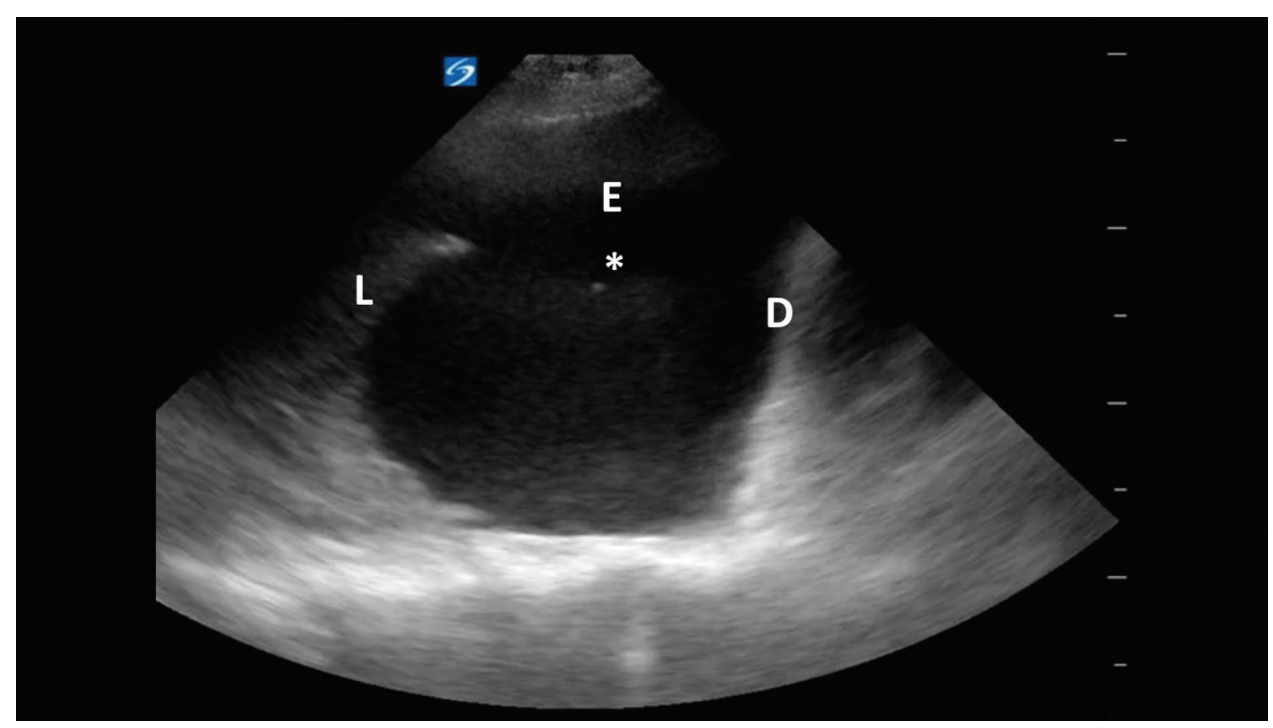

Figure 2. A sagittal ultrasound image of a complex non-septated pleural effusion (E) with a single echogenic particle $\left(^{*}\right)$, lung (L) and diaphragm (D) identified. It is worth noting that echogenic particles within pleural fluid are best seen on video and not in a still image. 


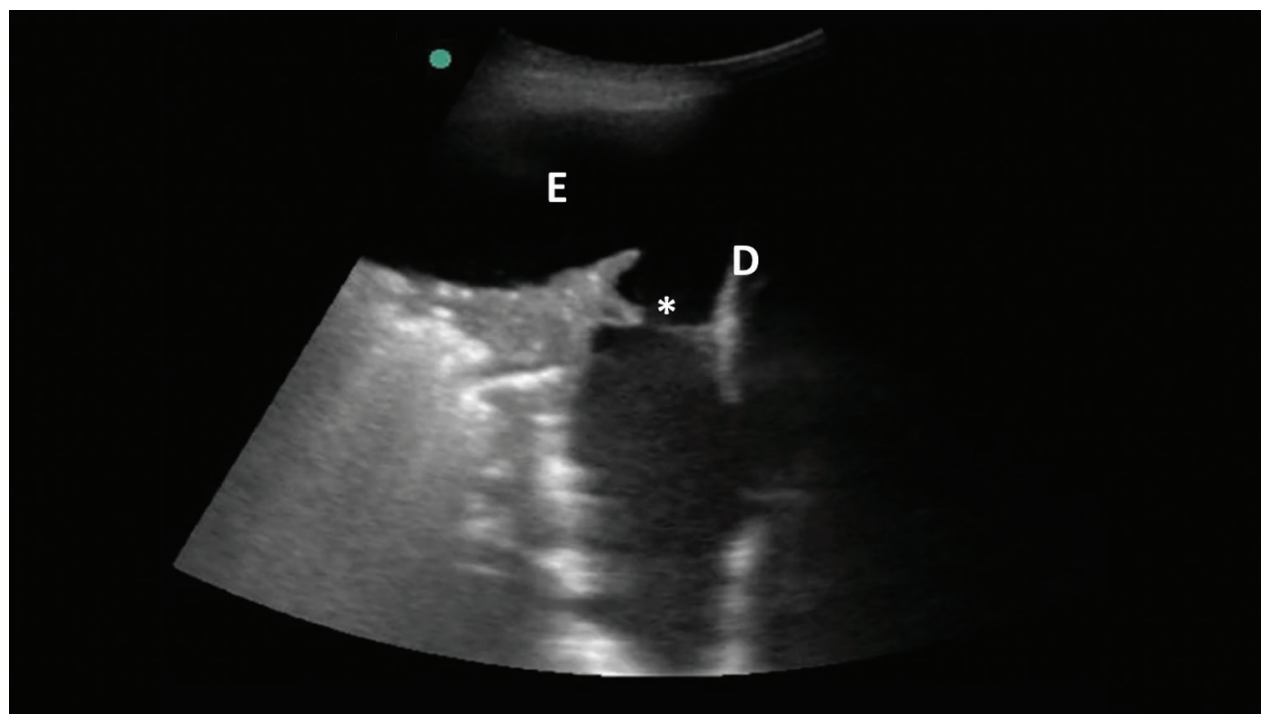

Figure 3. A sagittal ultrasound image of a complex septated pleural effusion (E) as evidenced by the presence of a fibrin strand (*) tethering the lung to the diaphragm (D).

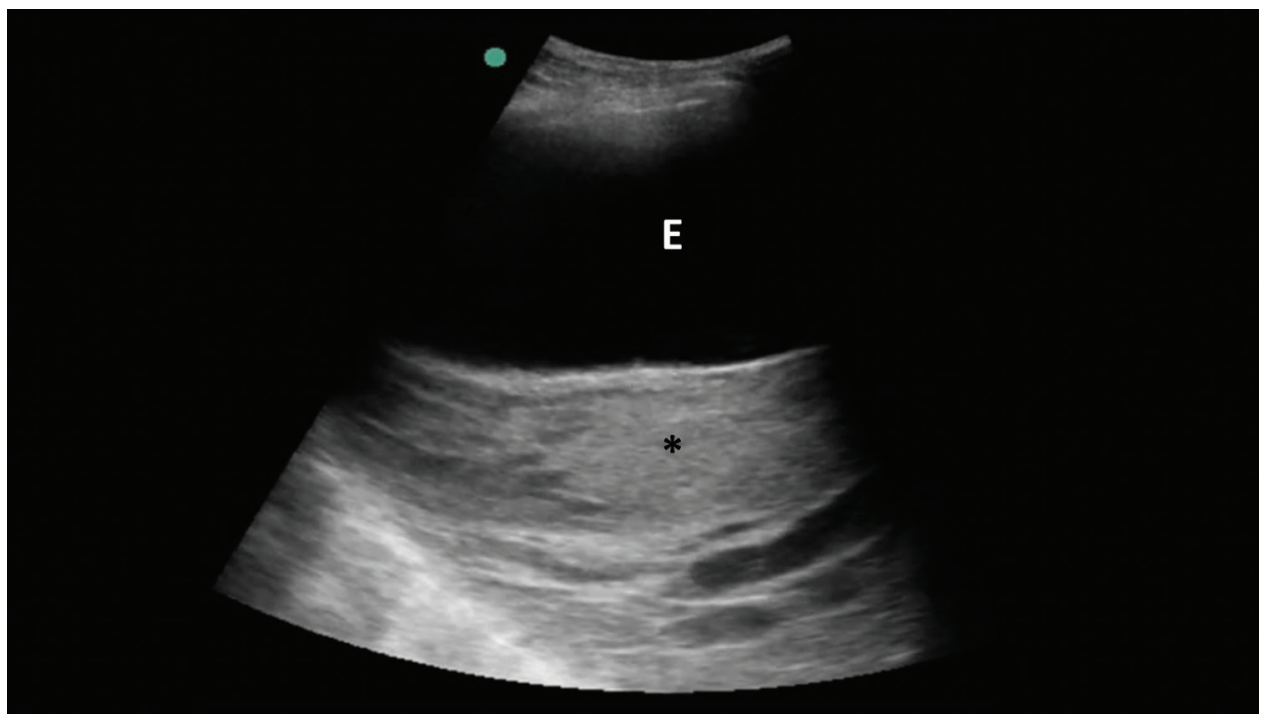

Figure 4. A sagittal ultrasound image of the superior right thorax demonstrating an anechoic pleural effusion (E) in addition to a homogenous echogenic pleural effusion (*).

of transudative pleural effusions. ${ }^{11,13}$ The presence of pleural fluid that is echogenic (bright) is referred to as a homogenous echogenic pattern and is most common in hemothorax (malignant or traumatic) and empyema. ${ }^{11}$

Sonographic abnormalities of the pleura such as thickening or nodules are features of exudative and malignant pleural effusions. Pleural thickening over $3 \mathrm{~mm}$ suggests an exudative effusion; whereas thickening over $1 \mathrm{~cm}$ is predominantly seen in malignant effusions. ${ }^{11}$ Pleural nodules or masses are highly associated with malignant effusions, but can also be found in tuberculosis, pleural space fibrosis (fibrothorax) and asbestosis. ${ }^{14}$ In one study, ultrasound demonstrated good sensitivity and specificity (sensitivity $80 \%$, specificity $84 \%$ ) in differentiating malignant from non-malignant pleural effusions. ${ }^{15}$ 
As described above, features of an exudative and malignant effusions arise from the sonographic abnormalities of the pleural fluid and pleura. Depending on the abnormality present, a general internist can then refine the pre-test probability of an exudative or malignant effusion and adjust clinical management accordingly. While point of care ultrasound can find signs suggesting an exudative or malignant effusion, it should not be used as sole modality to exclude either condition.

\section{Effusion Size and Procedural Guidance}

Based on its appearance on ultrasound, the size of a pleural effusion can be visually described as small, moderate or large; alternatively the volume of a pleural effusion can be estimated using several mathematical models, the simplest of which involves measuring the distance from the visceral to the parietal pleura, referred to as the maximal expiratory interpleural distance (MEPD) ${ }^{16-18}$ The MEPD is greatest at the most dependent aspect of the collection and is therefore measured at the posterior axillary line in supine patients and at the mid-scapular line in upright patients. For a rapid estimate of pleural fluid volume at the bedside, a MEPD of $5 \mathrm{~cm}$ measured in the transverse plane predicts an effusion volume of greater than $500 \mathrm{~mL}$ with good sensitivity and moderate specificity (sensitivity $83-100 \%$, specificity $67-90 \%))^{2,19}$

Consensus guidelines support the use of ultrasound guidance for all diagnostic pleural aspirations due to improved success rate and decreased risk of complications. ${ }^{20}$ The most common complication of thoracentesis is pneumothorax, occurring on average in $6 \%$ of procedures. ${ }^{3}$ Ultrasound guidance reduced the rate of pneumothorax by $16.3 \%$ in one study reviewing nearly twenty-thousand procedures, and by $43 \%$ in an earlier published systemic review and meta-analysis. ${ }^{3,4}$ The rate of hemorrhage was reduced by $38.7 \%$ with the use of ultrasound guidance. ${ }^{4}$

As indicated earlier, point of care ultrasound can be used to estimate the size of a pleural effusion, enabling identification of pleural effusions suitable for thoracentesis. Due to lower rates of complications, ultrasound guidance is essential when preforming a diagnostic or therapeutic thoracentesis.

\section{Limitations}

Limitations of point of care can be divided into four areas: operator, access, and technical limitations. Point of care ultrasound, by nature is a heavily operator dependent technique that utilizes a non-comprehensive ultrasound examination to answer a specific clinical question. Operator inexperience with either image acquisition or interpretation can lead to false positive and negative findings, highlighting the need for quality assurance and adequate training. Comprehensive and detailed examination with chest radiographs or chest CT may uncover additional abnormalities outside the area of examination. Access to and portability of ultrasound machines on a general medical ward may be challenging in contrast to the ubiquity of ultrasound machines in emergency rooms and intensive care units. Patient factors such as morbid obesity, chest bandages and positioning can lead to a non-diagnostic examination. Lastly, pleural and chest wall tumours, lymphoma and neurogenic tumours can mimic the appearance of a pleural effusion. ${ }^{21}$

\section{Summary}

The use of point of care ultrasound offers many advantages to the general internist who commonly diagnoses and manages pleural effusions as part of their practice. It provides superior diagnostic performance and additional diagnostic information when compared to physical exam or chest radiography alone, and is associated with increased procedural success and reduced rates of complications during thoracentesis.

\section{Learning Pearls}

- POCUS has superior sensitivity and specificity in detecting pleural effusions when compared to physical exam or chest radiography

- POCUS can detect features of exudative and malignant pleural effusions

- POCUS examination of the lungs in experienced hands can be performed and interpreted within minutes at the bedside

- POCUS guidance should be used for all diagnostic pleural aspirations due to improved success rate and decreased risk of complications

\section{References}

1. Puchalski JT. Mortality of hospitalized patients with pleural effusions. J Pulmonar Respirator Med 2014;04(03).

2. Vignon P, Chastagner C, Berkane V, et al. Quantitative assessment of pleural effusion in critically ill patients by means of ultrasonography. Crit Care Med 2005;33(8):1757-63.

3. Gordon CE. Pneumothorax following thoracentesis. Arch Intern Med 2010;170(4):332.

4. Patel PA, Ernst FR, Gunnarsson CL. Ultrasonography guidance reduces complications and costs associated with thoracentesis procedures. J Clin Ultrasound 2012;40(3):135-41.

5. Yousefifard M, Baikpour M, Ghelichkhani P, et al. Screening performance characteristic of ultrasonography and radiography in detection of pleural effusion; a meta-analysis. Emerg (Tehran) 2016;4(1):1-10.

6. Schleder S, Dornia C, Poschenrieder F, et al. Bedside diagnosis of pleural effusion with a latest generation hand-carried ultrasound device in intensive care patients. Acta Radiologica 2012;53(5):556-60.

7. Begot E, Grumann A, Duvoid T, et al. Ultrasonographic identification and semiquantitative assessment of unloculated pleural effusions in critically ill patients by residents after a focused training. Intens Care Med 2014;40(10):1475-80.

8. Wong CL, Holroyd-Leduc J, Straus SE. Does this patient have a pleural effusion? JAMA 2009;301(3):309-17. 
9. Lichtenstein D, Goldstein I, Mourgeon E, Cluzel P, Grenier P, Rouby J-J. Comparative diagnostic performances of auscultation, chest radiography, and lung ultrasonography in acute respiratory distress syndrome. Anesthesiology 2004;100(1):9-15.

10. Tayal VS, Nicks BA, Norton HJ. Emergency ultrasound evaluation of symptomatic nontraumatic pleural effusions. Am J Emerg Med 2006;24(7):782-6.

11. Yang PC, Luh KT, Chang DB, Wu HD, Yu CJ, Kuo SH. Value of sonography in determining the nature of pleural effusion: analysis of 320 cases. Am J Roentgenol 1992;159(1):29-33.

12. Chian C-F, Su W-L, Soh L-H, Yan H-C, Perng W-C, Wu C-P. Echogenic swirling pattern as a predictor of malignant pleural effusions in patients with malignancies. Chest 2004;126(1):129-34.

13. Chen H-J, Tu C-Y, Ling S-J, et al. Sonographic appearances in transudative pleural effusions: not always an anechoic pattern. Ultrasound Med Biol 2008;34(3):362-9.

14. Görg C, Restrepo I, Schwerk WB. Sonography of malignant pleural effusion. Eur Radiol 1997;7(8):1195-8.

15. Bugalho A, Ferreira D, Dias SS, et al. The diagnostic value of transthoracic ultrasonographic features in predicting malignancy in undiagnosed pleural effusions: a prospective observational study. Respiration 2014;87(4):270-8.

16. Balik M, Plasil P, Waldauf $P$, et al. Ultrasound estimation of volume of pleural fluid in mechanically ventilated patients. Intensive Care Med 2006;32(2):318-21.

17. Usta E, Mustafi M, Ziemer G. Ultrasound estimation of volume of postoperative pleural effusion in cardiac surgery patients. Interact Cardio Thoracic Surg 2010;10(2):204-7.

18. Remérand F, Dellamonica J, Mao Z, et al. Multiplane ultrasound approach to quantify pleural effusion at the bedside. Intensive Care Med 2010;36(4):656-64.

19. Roch A, Bojan M, Michelet P, et al. Usefulness of ultrasonography in predicting pleural effusions $>500 \mathrm{~mL}$ in patients receiving mechanical ventilation. Chest 2005;127(1):224-32.

20. Havelock T, Teoh R, Laws D, Gleeson F, BTS Pleural disease guideline group. pleural procedures and thoracic ultrasound: British Thoracic Society Pleural Disease Guideline 2010. Thorax 2010;65 Suppl 2:ii61-76.

21. McLoud TC, Flower CD. Imaging the pleura: sonography, CT, and MR imaging. Am J Roentgenol 1991;156(6):1145-53. 